\title{
Eradication of singularities in the next-to-leading order RG evolution for the $\Delta S=1$ effective Hamiltonian with 3 quark flavours
}

\section{David H. Adams*}

Department of Physics and Astronomy, Seoul National University, Seoul, 151-747, South Korea E-mail: dadams@phya.snu.ac.kr

\section{Weonjong Lee}

Frontier Physics Research Division and Center for Theoretical Physics,

Department of Physics and Astronomy, Seoul National University, Seoul, 151-747, South Korea E-mail: wleelphya.snu.ac.kr

\begin{abstract}
We consider the renormalization group (RG) evolution for the operators in the $\Delta S=1$ effective Hamiltonian with 3 active quark flavors, which is needed in the numerical analysis of data sets for $\varepsilon^{\prime} / \varepsilon$ calculated in lattice QCD. Singularities are present in the original solution of Buras et al. at next-to-leading order (NLO). We show how these can be eradicated through a method of analytic continuation to obtain the correct finite solution in this case. Furthermore, we trace the origin of the singularities to a breakdown of the approach of Buras et al. in the 3 flavour case, and show how it can be rectified so that singularitites are absent from the beginning.
\end{abstract}

The XXV International Symposium on Lattice Field Theory

July 30-4 August 2007

Regensburg, Germany

\footnotetext{
* Speaker.
} 


\section{Introduction}

Weak $\Delta S=1$ decays of hadrons can be described in the framework of the Standard Model by an effective Hamiltonian $\mathscr{H}_{\text {eff }}$ obtained by integrating out the degrees of freedom associated with heavy quarks and gauge bosons [1, 2, 3, 4, 5]. It governs interesting aspects of kaon physics; in particular the direct CP violation parameter $\varepsilon^{\prime} / \varepsilon$ and the $\Delta I=1 / 2$ rule. The effective Hamiltonian has the form

$$
\mathscr{H}_{e f f}=\frac{G_{F}}{\sqrt{2}} \sum_{i} C_{i}(\mu, g, e) Q_{i}(\mu, g, e)
$$

where $C_{i}(\mu, g, e)$ denotes the Wilson coeffi cients, the $Q_{i}(\mu, g, e)$ 's are a basis of renormalized 4fermion operators contributing to the effective Hamiltonian, $\mu$ is the scale, $g$ the strong coupling and $e$ the EM coupling. We take the basis operators $Q_{i}$ to be the ones specifi ed by Buras et al. in Section 2 of Ref.[1]. There are 10 of these and their origins are as follows: $Q_{1-2}$ are from $W$-exchange, $Q_{3-6}$ are from QCD penguin diagrams while $Q_{7-10}$ are from electroweak penguin diagrams.

The energy scale in kaon decays is about $500 \mathrm{MeV}$, so the hadronic matrix elements of $\mathscr{H}_{\text {eff }}$ are dominated by the strong interaction and must be calculated non-perturbatively. The lattice approach is the only possibility for doing this at present, and is currently being used; see Ref.'s [6, 7, 8, 9]. The procedure for calculating the matrix elements from the lattice can be summarized as follows. First one calculates the matrix elements of the appropriate lattice operators $Q_{j}^{\text {latt }}(a)$. Next one matches to the continuum operators at some scale $q^{*} \approx 1 / a$ in a continuum renormalization scheme, e.g., Naive Dimensional Regularization (NDR):

$$
Q_{i}\left(q^{*}\right)=z_{i j}\left(q^{*} a\right) Q_{j}^{\text {latt }}(a)
$$

The matching factors $z_{i j}\left(q^{*} a\right)$ can be calculated in perturbation theory [12]; they involve $\log \left(q^{*} a\right)$ so the continuum scale $q^{*}$ must be close to $1 / a$ to avoid large logs. Finally, one uses RG evolution to run the continuum operators $Q_{i}(\mu)$ from the scale $\mu=q^{*}$ down to $\mu=m_{c}$ (the charm quark mass) where they can be combined with the known Wilson coeffi cients ${ }^{1}$ at the scale $\mu=m_{c}$ to get the matrix elements of $\mathscr{H}_{\text {eff }}{ }^{2}$

The RG evolution operator required in the fi nal step above has been calculated up to nextto-leading order (NLO) by Buras et al. [1]. However, the expression obtained there contains singularities in the case where there are 3 active quark fhvors. Therefore it cannot be used for extracting the matrix elements from lattice QCD simulations with $N_{f}=2+1$ dynamical fermion fhvors. This is a major problem since unquenched lattice simulations with $2+1$ sea quark flavors are currently underway with a variety of fermion discretizations (see, e.g., [10] and references therein) and will be addressing kaon physics such as $\varepsilon^{\prime} / \varepsilon$ and the $\Delta I=1 / 2$ rule in the coming future. It is therefore imperative to deal with the singularity problem in the NLO expression for the RG evolution operator. The full RG evolution operator is known to be singularity-free, so the singularity in the NLO expression in the 3 fluvor case should be a removable artifact. In this paper

\footnotetext{
${ }^{1}$ The Wilson coeffi cients are all known up to NLO in peturbation theory [1]].

${ }^{2}$ Note that $\mathscr{H}_{\text {eff }}$ itself is independent of the scale, so we can combine the operators and Wilson coeffi cients at any chosen scale to determine its matrix elements.
} 
we review the solution to this problem given recently in Ref.[11]. The singularity is eradicated by a method of analytic continuation to obtain the correct fi nite NLO expression for the RG operator in the 3 flavor case. Furthermore, we trace the origin of the singularities in the work of Buras et al. to a breakdown of their approach in the 3 flavor case, and show how it can be rectifi ed so that singularities are absent from the beginning.

\section{Review of RG evolution and the singularity problem at NLO}

The running of the $Q_{i}(\mu)$ 's and $C_{i}(\mu)$ 's is governed by the RG evolution equation resulting from $\frac{d}{d \mu} \mathscr{H}_{\text {eff }}=0$. Combining the operators and Wilson coeffi cients into vectors $\vec{Q}$ and $\vec{C}$ respectively, the evolution is given by $10 \times 10$ matrices acting on these vectors. The evolution of $\vec{Q}(\mu)$ is obviously inverse to that of $\vec{C}(\mu)$ so it suffices to determine the latter, which is what we do in the following. The running of the EM coupling $e$ is negligible over the range of scales that are relevant for the present considerations, so we treat it as constant in the following, as was done in Ref.[1]].

The RG equation for $\vec{C}(\mu)$ is

$$
\left[\mu \frac{\partial}{\partial \mu}+\beta(g, e) \frac{\partial}{\partial g}\right] \vec{C}=\gamma^{T}(g, e) \vec{C}
$$

where $\gamma(g, e)$ is the $10 \times 10$ anomalous dimension matrix (given below) and $\beta(g, e)$ is the betafunction, given by

$$
\beta(g, e)=-\beta_{0} \frac{g^{3}}{16 \pi^{2}}-\beta_{1} \frac{g^{5}}{\left(16 \pi^{2}\right)^{2}}-\beta_{1 e} \frac{e^{2} g^{3}}{\left(16 \pi^{2}\right)^{2}}+\ldots
$$

with

$$
\beta_{0}=11-\frac{2}{3} f, \quad \beta_{1}=102-\frac{38}{3} f, \quad \beta_{1 e}=-\frac{8}{9}\left(u+\frac{d}{4}\right)
$$

where $u$ and $d$ denote the number of active $u$ - and $d$-type flavors, respectively, and $f=u+d$ $\left(=N_{f}\right)$ is the total number of active fhvors.

From the RG equation (2.1) the running of $\vec{C}(\mu)$ is found to be given by

$$
\vec{C}\left(m_{1}\right)=U\left(m_{1}, m_{2}\right) \vec{C}\left(m_{2}\right)
$$

where the evolution matrix is

$$
U\left(m_{1}, m_{2}\right)=T_{g} \exp \left(\int_{g\left(m_{2}\right)}^{g\left(m_{1}\right)} d g^{\prime} \frac{\gamma^{T}\left(g^{\prime}, e\right)}{\beta\left(g^{\prime}, e\right)}\right)
$$

with the dependence $g=g(m)$ specifi ed by $m_{d m}^{d g}=\beta(g, e)$. Here $T_{g}$ denotes $g$-ordering; it is required since generally $\left[\gamma\left(g_{1}\right), \gamma\left(g_{2}\right)\right] \neq 0$ for $g_{1} \neq g_{2}$.

To evaluate $U\left(m_{1}, m_{2}\right)$ we need to know the anomalous dimension matrix $\gamma(g, e)$. It is determined from the renormalization constant matrix relating the bare and renormalized operators: $Q_{i}^{(0)}=Z_{i j}(\mu) Q_{j}(\mu)$ and

$$
\gamma(g, e)=Z^{-1} \frac{d}{d \log \mu} Z
$$


This depends on $g$ and $e$ through $\alpha_{s}=\frac{g^{2}}{4 \pi}$ and $\alpha=\frac{e^{2}}{4 \pi}$, and has been calculated perturbatively up to 2 loops in the NDR scheme, whereby the matrices in the following expansions have been determined (see [1] and the references therein):

$$
\gamma(g, e)=\gamma_{s}\left(g^{2}\right)+\frac{\alpha}{4 \pi} \Gamma\left(g^{2}\right)+O\left(\alpha^{2}\right)
$$

where the pure QCD part is

$$
\gamma_{s}\left(g^{2}\right)=\frac{\alpha_{s}}{4 \pi} \gamma_{s}^{(0)}+\frac{\alpha_{s}^{2}}{(4 \pi)^{2}} \gamma_{s}^{(1)}+\ldots
$$

and the leading order QED correction in (2.7) is specifi ed by

$$
\Gamma\left(g^{2}\right)=\gamma_{e}^{(0)}+\frac{\alpha_{s}}{4 \pi} \gamma_{s e}^{(1)}+\ldots
$$

For later use we note that

$$
\begin{aligned}
\frac{\gamma(g, e)}{\beta(g, e)} & =-\frac{4 \pi}{\beta_{0} g^{3}}\left[\begin{array}{l}
\alpha_{s} \gamma_{s}^{(0)}+\frac{\alpha_{s}^{2}}{4 \pi}\left(\gamma_{s}^{(1)}-\frac{\beta_{1}}{\beta_{0}} \gamma_{s}^{(0)}\right) \\
+\alpha\left(\gamma_{e}^{(0)}+\frac{\alpha_{s}}{4 \pi}\left(\gamma_{s e}^{(1)}-\frac{\beta_{1}}{\beta_{0}} \gamma_{e}^{(0)}-\frac{\beta_{1 e}}{\beta_{0}} \gamma_{s}^{(0)}\right)+O\left(\alpha_{s}^{2}\right)\right)+O\left(\alpha^{2}\right)
\end{array}\right] \\
& =-\frac{\gamma_{s}^{(0)}}{\beta_{0} g}+O(g)+O(\alpha)
\end{aligned}
$$

The expansion of the evolution operator in the EM coupling takes the form

$$
U\left(m_{1}, m_{2}\right)=U_{s}\left(m_{1}, m_{2}\right)+\frac{\alpha}{4 \pi} R\left(m_{1}, m_{2}\right)+O\left(\alpha^{2}\right)
$$

where the pure QCD evolution is

$$
U_{s}\left(m_{1}, m_{2}\right)=T_{g} \exp \left(\int_{g\left(m_{2}\right)}^{g\left(m_{1}\right)} d g^{\prime} \frac{\gamma_{s}^{T}\left(g^{\prime}\right)}{\beta_{s}\left(g^{\prime}\right)}\right)
$$

and the leading additional contribution to the QCD evolution in the presence of EM interactions in (2.12) is given by (see [1])

$$
R\left(m_{1}, m_{2}\right)=\int_{g\left(m_{2}\right)}^{g\left(m_{1}\right)} d g^{\prime} \frac{U_{s}\left(m_{1}, m^{\prime}\right) \Gamma^{T}\left(g^{\prime}\right) U_{s}\left(m^{\prime}, m_{2}\right)}{\beta_{s}\left(g^{\prime}\right)}
$$

where $g^{\prime}=g^{\prime}\left(m^{\prime}\right)$. The EM contribution to the beta-function has been ignored in (2.13)-(2.14): $\beta_{s}(g)=\beta(g, 0)$ so the expressions are valid when the $\beta_{1 e}$ term in $(2.2)$ is dropped, which is a justifi able approximation made in Ref.[1]. However, the generalization of the NLO expressions for the evolution matrix to the case where the $\beta_{1 e}$ term is not dropped is straightforward: In light of (2.10) it can be obtained simply by replacing $\gamma_{s e}^{(1)} \rightarrow \gamma_{s e}^{(1)}-\frac{\beta_{1 e}}{\beta_{0}} \gamma_{s}^{(0)}$ in the relevant expressions [11].

In the remainder of this paper we restrict our attention to the pure QCD evolution $U_{s}\left(m_{1}, m_{2}\right)$ which is where the aforementioned singularity problem arises at NLO in the 3 flavor case. Once the singularity is eradicated, the new fi nite expression needs to be used in the NLO evaluation of (2.14) for $R\left(m_{1}, m_{2}\right)$. We omit that part here, referring to Ref.[11] for the details and result. 
We begin by recalling the leading logarithmic approximation to $U_{s}\left(m_{1}, m_{2}\right)$ : it is obtained by keeping only the leading contribution to $\frac{\gamma_{s}^{T}\left(g^{\prime}\right)}{\beta_{s}\left(g^{\prime}\right)}$ in the integrand in (2.13), which is shown in (2.11). It is useful to diagonalize $\gamma_{s}^{(0) T}$ :

$$
\gamma_{D}^{(0)}=V^{-1} \gamma_{s}^{(0) T} V \quad \text { diagonal matrix }
$$

then the leading logarithmic approximation is found to be

$$
U_{s}^{(0)}\left(m_{1}, m_{2}\right)=V\left(\frac{\alpha_{s}\left(m_{2}\right)}{\alpha_{s}\left(m_{1}\right)}\right)^{\gamma_{D}^{(0)} / 2 \beta_{0}} V^{-1}
$$

The NLO contribution to the evolution matrix can now be found starting from the ansatz

$$
U_{s}\left(m_{1}, m_{2}\right)=\left(1+\frac{\alpha_{s}\left(m_{1}\right)}{4 \pi} J\right) U_{s}^{(0)}\left(m_{1}, m_{2}\right)\left(1-\frac{\alpha_{s}\left(m_{2}\right)}{4 \pi} J\right)
$$

and using the RG equation for $U_{s}\left(m_{1}, m_{2}\right)$ (given by (2.1) with $\beta(g, e)$ and $\gamma(g, e)$ replaced by $\beta_{s}(g)$ and $\gamma_{s}(g)$, respectively) to derive an equation for $J$. From solving this equation at lowest order in $\alpha_{s}$ Buras et al. find [1, [3]

$$
J=V S V^{-1}
$$

where

$$
S_{i j}=\delta_{i j} \gamma_{i}^{(0)} \frac{\beta_{1}}{2 \beta_{0}^{2}}-\frac{G_{i j}}{2 \beta_{0}\left(1+a_{i}-a_{j}\right)}
$$

with

$$
G=V^{-1} \gamma_{s}^{(1)} V \quad, \quad a_{i}=i^{\prime} \text { th diagonal element of } \gamma_{D}^{(0)} / 2 \beta_{0}
$$

From (2.19) we see that the resulting NLO expression for the evolution matrix has a singularity if $1+a_{i}-a_{j}=0$. As mentioned in [1], this happens for $(i, j)=(8,7)$ when there are 3 active quark fhvors $(f=3)$.

\section{Solution of the singularity problem via analytic continuation}

The expression (2.17) can be written up to NLO as

$$
U_{s}^{N L O}\left(m_{1}, m_{2}\right)=U_{s}^{(0)}\left(m_{1}, m_{2}\right)+\frac{1}{4 \pi} V A\left(m_{1}, m_{2}\right) V^{-1}
$$

where

$$
V A\left(m_{1}, m_{2}\right) V^{-1}=\alpha_{s}\left(m_{1}\right) J U_{s}^{(0)}\left(m_{1}, m_{2}\right)-\alpha_{s}\left(m_{2}\right) U_{s}^{(0)}\left(m_{1}, m_{2}\right) J
$$

Inserting the expression (2.16) for $U_{s}^{(0)}\left(m_{1}, m_{2}\right)$ leads to

$$
A_{i j}\left(m_{1}, m_{2}\right)=S_{i j}\left[\alpha_{1}\left(\frac{\alpha_{2}}{\alpha_{1}}\right)^{a_{j}}-\alpha_{2}\left(\frac{\alpha_{2}}{\alpha_{1}}\right)^{a_{i}}\right]
$$


where $\alpha_{k} \equiv \alpha_{s}\left(m_{k}\right)$ for $k=1,2$. In the singular case $(f=3, i=8, j=7)$ we now regularize $S_{i j}$ by replacing

$$
a_{j} \rightarrow a_{j}+\varepsilon
$$

in (2.19). The regularized quantity then becomes $S_{i j}=\left(\frac{G_{i j}}{2 \beta_{0}}\right) \frac{1}{\varepsilon}$. Inserting this into (3.3), and making the same regularization (3.4) there, we find

$$
\begin{aligned}
A_{i j}\left(m_{1}, m_{2}\right) & =\left(\frac{G_{i j}}{2 \beta_{0}}\right) \frac{1}{\varepsilon}\left[\alpha_{1}\left(\frac{\alpha_{2}}{\alpha_{1}}\right)^{a_{j}+\varepsilon}-\alpha_{2}\left(\frac{\alpha_{2}}{\alpha_{1}}\right)^{a_{i}}\right] \\
& =\left(\frac{G_{i j}}{2 \beta_{0}}\right) \frac{1}{\varepsilon} \alpha_{2}\left(\frac{\alpha_{2}}{\alpha_{1}}\right)^{a_{i}}\left[\varepsilon \log \left(\frac{\alpha_{2}}{\alpha_{1}}\right)+O\left(\varepsilon^{2}\right)\right] \\
& \stackrel{\varepsilon \rightarrow 0}{=}\left(\frac{G_{i j}}{2 \beta_{0}}\right) \alpha_{2}\left(\frac{\alpha_{2}}{\alpha_{1}}\right)^{a_{i}} \log \left(\frac{\alpha_{2}}{\alpha_{1}}\right)
\end{aligned}
$$

Thus a fi nite expression is obtained in the limit where the regularization is lifted. Substituting this into (3.1) we get a fi nite NLO expression for the evolution operator. This solution of the singularity problem clearly amounts to analytic continuation of the evolution operator (regarded as a function of the matrix $\left.\gamma_{s}^{(0)}\right)$.

\section{Understanding the "singularity" from first principles}

In the following it is convenient to use the notation $U_{s}\left(g_{1}, g_{2}\right)$ rather than $U_{s}\left(m_{1}, m_{2}\right)$; this is justifi ed since the dependence on $m_{1}, m_{2}$ enters exclusively through $g\left(m_{1}\right), g\left(m_{2}\right)$.

By general arguments the full evolution operator (for pure QCD) can be written as

$$
U_{s}\left(g_{1}, g_{2}\right)=\left(1+\frac{g_{1}^{2}}{16 \pi^{2}} J\left(g_{1}\right)\right) U_{s}^{(0)}\left(g_{1}, g_{2}\right)\left(1+\frac{g_{2}^{2}}{16 \pi^{2}} J\left(g_{2}\right)\right)^{-1}
$$

Using the RG equation

$$
\frac{d}{d g} U_{s}\left(g, g_{0}\right)=\frac{\gamma_{s}^{T}(g)}{\beta_{s}(g)} U_{s}\left(g, g_{0}\right)
$$

to derive an equation for $J(g)$ at leading order, one fi nds different equations in the "singular" case $1+a_{i}-a_{j}=0$ and "non-singular" case [11].

In the non-singular case, taking $J(g)=J+O(g)$ leads to a consistent solution for the constant matrix $J$. This is the solution found by Buras et al. that we reviewed in $\S 2$. On the other hand, in the singular case, the ansatz $J(g)=J+O(g)$ does not admit a consistent solution. In fact, it turns out that $S_{i j}(g)=\left(V^{-1} J(g) V\right)_{i j}$ must diverge for $g \rightarrow 0$. Specifi cally, in the singular case $1+a_{i}-a_{j}=0$ we found in Ref.[1], that the leading order equation for $S_{i j}(g)$ is as follows:

$$
\beta_{0} g \frac{d}{d g} S_{i j}(g)=-G_{i j}
$$

The solution is

$$
S_{i j}(g)=-\frac{G_{i j}}{\beta_{0}} \log (g)+c_{i j}=-\frac{G_{i j}}{2 \beta_{0}} \log \left(\alpha_{s}\right)+c_{i j}^{\prime}
$$


where $c_{i j}$ is an undetermined integration constant and $c_{i j}^{\prime}=c_{i j}-\frac{G_{i j}}{2 \beta_{0}} \log (4 \pi)$. Thus $S_{i j}(g)$ diverges for $g \rightarrow 0$ as claimed. Note however that $g^{2} S_{i j}(g)$ vanishes for $g \rightarrow 0$, implying that $g^{2} J(g)$ vanishes in this limit as it should in order for (4.1) to reduce to $U_{s}^{(0)}\left(m_{1}, m_{2}\right)$ in the small $g$ limit. We also note that $S_{i j}(g)$ is actually a function of $g^{2}$ (or $\alpha_{s}$ ) as it should be. Finally, our previous expression (3.5) for $A_{i j}\left(m_{1}, m_{2}\right)$ is readily reproduced from (4.4) (see [11]), ${ }^{3}$ so the fi rst principles solution of the singularity problem presented here agrees as it should with the fi nite NLO result for the evolution operator obtained via analytic continuation in the previous section.

\section{Conclusions}

We have eradicated the singularities in the original solution of Buras et al. to get a finite expression for the RG evolution matrix at NLO in the 3 flavor case. This is essential for being able to evaluate the matrix elements of the $\Delta S=1$ effective Hamiltonian from the lattice with $2+1$ sea quark fhavors.

The breakdown of the ansatz $J(g)=J+O(g)$ in Buras et al.'s approach, and the rectifi cation discussed here, illustrate some general subtleties to bear in mind when evaluating evolution operators in general.

\section{Acknowledgements}

This research is supported by the KICOS international cooperative research program (KICOS grant K20711000014-07A0100-01410), by the KRF grant KRF-2006-312-C00497, by the BK21 program of Seoul National University, and by the DOE SciDAC-2 program.

\section{References}

[1] A. Buras, et al., Nucl. Phys. B 408 (1993) 209, [arXiv:hep-ph/9303284].

[2] G. Buchalla, et al., Rev. Mod. Phys. 68 (1996) 1125, [arXiv:hep-ph/9512380].

[3] A. Buras, et al., Nucl. Phys. B 370 (1992) 69.

[4] M. Ciuchini, E. Franco, G. Martinelli and L. Reina, Phys. Lett. B 301, 263 (1993) [arXiv:hep-ph/9212203].

[5] M. Ciuchini, E. Franco, G. Martinelli and L. Reina, Nucl. Phys. B 415, 403 (1994) [arXiv:hep-ph/9304257].

[6] T. Blum et al., Phys. Rev. D68 (2003) 114506, [arXiv:hep-lat/0110075].

[7] J. Noaki et al., Phys. Rev. D68 (2003) 014501, [arXiv:hep-lat/0108013].

[8] T. Bhattacharya et al., Nucl. Phys. B (Proc. Suppl.) 140 (2005) 169, [arXiv:hep-lat/0409046].

[9] W. Lee, Nucl. Phys. B (Proc. Suppl.) 128 (2004) 125, [arXiv:hep-lat/0310047].

[10] W. Lee, PoS LAT2006 (2006) 015, [arXiv:hep-lat/0610058].

[11] D. H. Adams and W. Lee, Phys. Rev. D75:074502 (2007) [arXiv:hep-lat/0701014].

[12] K. Hornbostel, G.P. Lepage, C. Morningstar, Phys. Rev. D67 (2003) 034023, [arXiv:hep-ph/0208224].

\footnotetext{
${ }^{3}$ The occurrences of the undetermined constant $c_{i j}^{\prime}$ from (4.4) are found to cancel out in $A_{i j}\left(m_{1}, m_{2}\right)$ [11].
} 